\title{
Practice-based Research Theme Issue and 2006 Highlights
}

\section{Thanks to 2006 Peer Reviewers}

In this issue, we thank and acknowledge by name the individuals who provided external peer reviews for the Fournal of the American Board of Family Medicine (7ABFM) in 2006 (pp. 235-238). Their invaluable service to advancing the science of primary care is in addition to the critical appraisal provided by our Editorial Board members and Associate Editors. Thank you!

\section{Practice-based Research Theme Issue}

This March-April 2007 issue is the second $7 A B F M$ theme issue dedicated to practice-based research; the first was published in January 2006. The current work of 11 practice-based research networks (PBRNs) is highlighted in this issue. Many of these contributions were presented in 2006 at either the Agency for Healthcare Research and Quality (AHRQ) National PBRN Research Conference in Bethesda, MD, or at the American Academy of Family Physicians' (AAFP) National Research Network (NRN) Convocation of Practices and Networks in Dallas, TX.

The eclectic group of papers in this special theme issue includes reports of original research and papers about PBRN processes. In 2 manuscripts, the AAFP NRN provides guidance for launching PBRN studies and for addressing the institutional review board challenges of multisite community-based research. The Oregon Rural Practice-Based Research Network (ORPRN) provides survey tools for characterizing a PBRN. The use of information technology is highlighted in papers from 5 networks, including the Electronic Primary Care Research Network (ePCRN), the Oklahoma Physicians Resource/Research Network (OKPRN), the Southern Primary-care Urban Research Network (SPUR-NET), the Indiana University Medical Group Research Network (ResNet), and the Central Texas Primary Care Research Network (CenTexNet).

Three PBRNs provide prevalence data for problems frequently addressed in primary care practice:
Research Involving Outpatient Settings Network (RIOS) explored tobacco use in vulnerable populations; HamesNet investigated patient perceptions of dysphagia; and the North Carolina Family Practice Research Network (NC-FP-RN) studied poor nutritional habits in primary care patients. Research findings related to patient safety and medical errors are reported by the High Plains Research Network (HPRN) and the AAFP NRN.

Finally, this theme issue contains 2 important essays that reflect on the future of practice-based research: George Rust and Lisa Cooper provide thoughtful consideration on how practice-based research can contribute to the elimination of health disparities, while Kevin Peterson outlines recent changes in the funding climate for practice-based research. The $7 A B F M$ is committed to disseminating research that can improve primary health care and recognizes the important work underway in PBRNs.

\section{Digital Object Identifiers}

The $7 A B F M$ is now a member of CrossRef (http:// www.crossref.org) and began assigning Digital Object Identifiers (DOIs) to each $7 A B F M$ article, as well as errata and letters to the editor, starting with the January-February 2007 issue. A DOI is a globally unique and persistent alphanumeric label for an instance of intellectual property, which allows users to locate items independently of their location on a particular Web site. The Digital Object Identifier System is supported by the International DOI Foundation, with information available at http://www.doi.org.

\section{Bibliographic Indexing}

The $7 A B F M$ is now listed in the Cumulative Index of Nursing and Allied Health Literature (CINAHL) database (www.cinahl.com).

\section{JABFM on the URM Journal List}

The $7 A B F M$ is now listed among the publications that follow the International Committee of Medical 
Journal Editor's Uniform Requirements for Manuscripts Submitted to Biomedical Journals (URM), available at http://www.icmje.org. See the URM journals list at http://www.icmje.org/jrnlist.html.

The URM provide useful guidelines for preparing and submitting a manuscript for publication. Authors are also encouraged to become familiar with the ICMJE policy statements that cover the following ethical domains in biomedical publishing:

\section{Ethical Considerations in the Conduct and Reporting of Research}

- Authorship and Contributorship

- Editorship

- Peer Review

- Conflicts of Interest

- Privacy and Confidentiality

- Protection of Human Subjects and Animals in Research
Publishing and Editorial Issues Related to

Publication in Biomedical Journals

- Obligation to Publish Negative Studies

- Corrections, Retractions, and "Expressions of Concern"

- Copyright

- Overlapping Publications

- Correspondence

- Supplements, Theme Issues, and Special Series

- Electronic Publishing

- Advertising

- Medical Journals and the General Media

- Obligation to Register Clinical Trials

Anne Victoria Neale, PhD, MPH, Deputy Editor Marjorie A. Bowman, MD, MPA, Editor Cynthia A. Rose, MBA, Publications Coordinator 